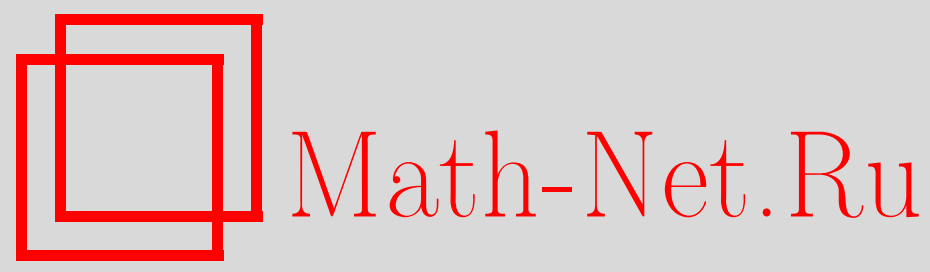

А. П. Солодов, Интегралы Хенстока и Мак-Шейна для банаховозначных функций, Матем. заметки, 1999, том 65, выпуск 6, 860-870

DOI: https://doi.org/10.4213/mzm1122

Использование Общероссийского математического портала Math-Net.Ru подразумевает, что вы прочитали и согласны с пользовательским соглашением http://www.mathnet.ru/rus/agreement

Параметры загрузки:

IP : 18.207 .199 .55

26 апреля 2023 г., $15: 13: 12$

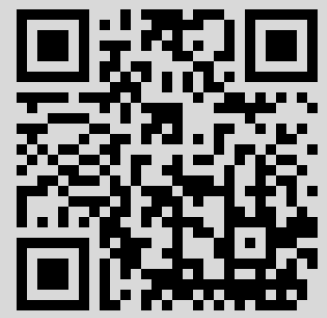




\title{
ИНТЕГРАЛЫ ХЕНСТОКА И МАК-ШЕЙНА ДЛЯ БАНАХОВОЗНАЧНЫХ ФУНКЦИЙ
}

\section{А. П. Солодов}

\begin{abstract}
В работе решается вопрос об эквивалентности $H L$-интеграла интегралу Хенстока в банаховых пространствах. Именно, доказывается, что справедливость леммы Сакса-Хенстока в банаховом пространстве равносильна его конечномерности.

Библиографоия: 11 названий.
\end{abstract}

В этой работе рассматриваются интегралы Хенстока и Мак-Шейна для функций, определенных на отрезке прямой и принимающих значения в банаховом пространстве. Эти интегралы, определяемые с помошью обобщенных сумм Римана, в действительном случае эквивалентны соответственно узкому интегралу Данжуа и интегралу Лебега (см. [1] и [2]). Значительная часть важных свойств этих интегралов, включая указанную эквивалентность, выводится из так называемой леммы Сакса-Хенстока (см. теорему 1.4). Однако, как показано в [3], эта лемма может не выполняться для банаховозначных функций. При этом в качестве примера для пространства значений в [3] берется пространство континуальной размерности. Возникает вопрос: для каких пространств лемма Сакса-Хенстока вьполняется. Ввиду важности леммы Сакса-Хенстока С. С. Као в [3] определил $H L$-интеграл, являюшийся сужением интеграла Хенстока, в котором выполнение условия этой леммы включено в определение.

В данной работеполностью решается вопрос о том, для каких банаховых пространств лемма Сакса-Хенстока выполняется для интеграла Хенстока. Другими словами, для каких пространств $H L$-интеграл эквивалентен интегралу Хенстока. А именно, здесь доказывается, что справедливость леммы Сакса-Хенстока в банаховом пространстве равносильна его конечномерности (см. теорему 3.1).

1. Основные определения и предварительные результаты. Пусть $X$ - банахово пространство, $\mathbb{R}$ - действительная прямая, $[a, b]$ - отрезок на прямой, $\mathscr{I}$ - совокупность всех отрезков, содержашихся в $[a, b]$. Разбиением $T$ отрезка $[a, b]$ назовем набор пар $\left(\xi_{k}, \Delta_{k}\right) \in[a, b] \times \mathscr{I}, k=1, \ldots, n$, обладаюший следуюшими свойствами:

a) отрезки $\Delta_{i}$ и $\Delta_{j}$ не перекрываются, если $i \neq j$;

б) $\bigcup_{k=1}^{n} \Delta_{k}=[a, b]$.

Если не требовать вьполнения свойства б), то такой набор будем назьвать подразбиением.

Работа выполнена при поддержке Российского фонда фундаментальных исследований, грант № 96-01-00332, и программы "Ведущие научные школы", грант № 96-15-96073. 
Напомним определения интегралов Хенстока и Мак-Шейна (см. [4], [5]). Пусть $\delta(\xi)-$ положительная функция, заданная на отрезке $[a, b]$.

ОпредЕлЕниЕ 1.1. Разбиение (подразбиение) $T$ отрезка $[a, b]$ называется $\delta$-согл $a$ сованным по Хенстоку, если каждая пара $(\xi, \Delta) \in T$ удовлетворяет условию

$$
\xi \in \Delta \subset(\xi-\delta(\xi), \xi+\delta(\xi)) .
$$

ОПрЕДЕЛЕниЕ 1.2. Разбиение (подразбиение) $T$ отрезка $[a, b]$ назьвается $\delta$-согл $a$ сованным по Мак-Шейну, если каждая пара $(\xi, \Delta) \in T$ удовлетворяет условию

$$
\Delta \subset(\xi-\delta(\xi), \xi+\delta(\xi)) \text {. }
$$

ОПРЕДЕЛЕНИЕ 1.3. Функция $f:[a, b] \rightarrow X$ назьвается интегрируемой по Хенстоку на отрезке $[a, b]$, если существует $I \in X$ со следуюшим свойством: для любого $\varepsilon>0$ найдется положительная функция $\delta(\xi)$ такая, что для любого $\delta$-согласованного по Хенстоку разбиения $T$ отрезка $[a, b]$ выполняется неравенство

$$
\left\|\sum_{T} f\left(\xi_{k}\right)\left|\Delta_{k}\right|-I\right\|<\varepsilon
$$

Вектор $I$ называется интегралом Хенстока функции $f$ по отрезку $[a, b]$ и записьвается в виде

$$
I=(H) \int_{a}^{b} f d x .
$$

Здесь $|P|$ обозначает меру множества $P$.

ОПРЕДЕЛЕнИЕ 1.4. Функция $f:[a, b] \rightarrow X$ называется интегрируемой по МакШейну на отрезке $[a, b]$, если существует $I \in X$ со следующим свойством: для любого $\varepsilon>0$ найдется положительная функция $\delta(\xi)$ такая, что для любого $\delta$-согласованного по Мак-Шейну разбиения $T$ отрезка $[a, b]$ вьполняется неравенство (1). Вектор $I$ называется интегралом Мак-Шейна функции $f$ по отрезку $[a, b]$ и записывается в виде

$$
I=(M) \int_{a}^{b} f d x .
$$

Следуюшие две теоремы представляют собой основные свойства интегралов Хенстока и Мак-Шейна. Их доказательство совершенно аналогично действительному случаю (см. [6]).

Tеорема 1.1. 1) Если функиия $f:[a, b] \rightarrow X$ интегрируема по Хенстоку (МакШейну) на отрезке $[a, b]$, то она интегрируема по Хенстоку (Мак-Шейну) и на любом отрезке $[c, d] \subset[a, b]$.

2) Пусть $c \in[a, b]$. Если функиия $f:[a, b] \rightarrow X$ интегрируема по Хенстоку (МакШейну) на отрезках $[a, c] u[c, b]$, то она интегрируема по Хенстоку (Мак-Шейну) и на отрезке $[a, b]$, причем

$$
\int_{a}^{b} f d x=\int_{a}^{c} f d x+\int_{c}^{b} f d x .
$$


Теорема 1.2. Если функиия $f:[a, b] \rightarrow X$ интегрируема по Мак-Шейну на отрезке $[a, b]$, то она интегрируема и по Хенстоку на отрезке $[a, b]$, при этом интеграль совпадают.

Теорема 1.1 позволяет ввести понятие первообразной для функции, интегрируемой по Хенстоку. В силу теоремы 1.2 оно будет включать в себя понятие первообразной для функции, интегрируемой по Мак-Шейну.

ОПРЕДЕЛЕНИЕ 1.5. Функция $F:[a, b] \rightarrow X$ назьвается первообразной для интегрируемой на $[a, b]$ по Хенстоку функции $f:[a, b] \rightarrow X$, если

$$
F(t)=\int_{a}^{t} f d x
$$

для всех $t \in[a, b]$.

Из теоремы 1.1 легко вытекает следующее утверждение.

ЛЕмма 1.1. Пусть функиия $f:[a, b] \rightarrow X$ интегрируема по Хенстоку (МакШейну) с первообразной $F:[a, b] \rightarrow X$. Тогда для любого $\varepsilon>0$ найдется положительная функиия $\delta(\xi)$ такая, что для любого $\delta$-согласованного по Хенстоку (Мак-Шейну) разбиения Т отрезка $[a, b]$ выполняется неравенство

$$
\left\|\sum_{T}\left(f\left(\xi_{k}\right)\left|\Delta_{k}\right|-F\left(\Delta_{k}\right)\right)\right\|<\varepsilon .
$$

Здесь $F(\Delta)$ обозначает приращение функиии $F$ на отрезке $\Delta$.

Приведенное ниже утверждение показьвает, что в лемме 1.1 разбиение может быть заменено на подразбиение.

ЛЕмма 1.2. Функиия $f:[a, b] \rightarrow X$ интегрируема по Хенстоку (Мак-Шейну) $c$ первообразной $F:[a, b] \rightarrow X$ тогда и только тогда, когда для любого $\varepsilon>0$ найдется положительная функиия $\delta(\xi)$ на $[a, b]$ такая, что для любого $\delta$-согласованного по Хенстоку (Мак-Шейну) подразбиения Т отрезка $[a, b]$ выполняется неравенство (2).

Доказательство леммы 1.2 можно найти в [7]. С ее помощью, как и в действительном случае (см. [6, с. 18, 105]), легко получить свойство непрерывности первообразной.

Теорема 1.3. Пусть функиия $f:[a, b] \rightarrow X$ интегрируема по Хенстоку (МакШейну) на отрезке $[a, b]$. Тогда ее первообразная $F:[a, b] \rightarrow X$ непрерывна.

Сформулируем теперь теорему, назьваемую леммой Сакса-Хенстока, справедливую в случае конечномерного пространства $X$.

Теорема 1.4. Пусть $X$ - конечномерное банахово пространство. Если функиия $f:[a, b] \rightarrow X$ интегрируема по Хенстоку (Мак-Шейну) и имеет первообразную $F:[a, b] \rightarrow X$, то для любого $\varepsilon>0$ найдется полохстельная функиия $\delta(\xi)$ такая, что для любого $\delta$-согласованного по Хенстоку (Мак-Шейну) подразбиения $T$ отрезка $[a, b]$ выполняется неравенство

$$
\sum_{T}\left\|f\left(\xi_{k}\right)\left|\Delta_{k}\right|-F\left(\Delta_{k}\right)\right\|<\varepsilon .
$$


ДокАЗАтЕльство. Случай $X=\mathbb{R}$ подробно разобран в $[6$, с. 17,104$]$. Идея доказательства состоит в том, что произвольное подразбиение $T$ разбивается на два: $T_{+}$ и $T_{-}$. Они определяются следуюшим образом: $(\xi, \Delta) \in T_{+}$, если $f(\xi)|\Delta|-F(\Delta) \geqslant 0$, $(\xi, \Delta) \in T_{-}$, если $f(\xi)|\Delta|-F(\Delta)<0$. Теперь теорема доказьвается с помощью леммы 1.2 .

Пусть $X=\mathbb{R}^{n}$. Тогда $f(t)=\left(f_{1}(t), f_{2}(t), \ldots, f_{n}(t)\right)$, где все $f_{i}$ - интегрируемые по Хенстоку (Мак-Шейну) функции, принимающие действительные значения и имеющие первообразные $F_{i}$. Фиксируем произвольное $\varepsilon>0$. Пользуясь справедливостью леммы Сакса-Хенстока для случая $X=\mathbb{R}$, выберем положительную функцию $\delta(\xi)$ так, чтобы для любого $\delta$-согласованного по Хенстоку (Мак-Шейну) подразбиения $T$ отрезка $[a, b]$ и для всех $i=1,2, \ldots, n$ выполнялись неравенства

$$
\sum_{T}\left|f_{i}\left(\xi_{k}\right)\right| \Delta_{k}\left|-F_{i}\left(\Delta_{k}\right)\right|<\frac{\varepsilon}{n} .
$$

Все нормы в $\mathbb{R}^{n}$ эквивалентны. Выбирая в качестве нормы сумму модулей координат, получим

$$
\begin{aligned}
\sum_{T}\left\|f\left(\xi_{k}\right)\left|\Delta_{k}\right|-F\left(\Delta_{k}\right)\right\| & =\sum_{T}\left(\sum_{i=1}^{n}\left|f_{i}\left(\xi_{k}\right)\right| \Delta_{k}\left|-F_{i}\left(\Delta_{k}\right)\right|\right) \\
& =\sum_{i=1}^{n}\left(\sum_{T}\left|f_{i}\left(\xi_{k}\right)\right| \Delta_{k}\left|-F_{i}\left(\Delta_{k}\right)\right|\right)<n \cdot \frac{\varepsilon}{n}=\varepsilon .
\end{aligned}
$$

Таким образом, теорема доказана (подробнее см. [3]).

2. $H L$-интеграл и $M L$-интеграл. Введем интегралы, в само определение которых входит требование выполнения леммы Сакса-Хенстока (см. [3], [8]).

ОПРЕДЕЛЕНИЕ 2.1. Функция $f:[a, b] \rightarrow X$ назьвается $H L$-интегрируемой на отрезке $[a, b]$, если существует функция $F:[a, b] \rightarrow X$ со следующим свойством: для любого $\varepsilon>0$ найдется положительная функция $\delta(\xi)$ такая, что для любого $\delta$-согласованного по Хенстоку разбиения $T$ отрезка $[a, b]$ выполняется неравенство (3).

ОПРЕДЕЛЕНИЕ 2.2. Функция $f:[a, b] \rightarrow X$ назьвается $M L$-интегрируемой на отрезке $[a, b]$, если существует функция $F:[a, b] \rightarrow X$ со следуюшим свойством: для любого $\varepsilon>0$ найдется положительная функция $\delta(\xi)$ такая, что для любого $\delta$-согласованного по Мак-Шейну разбиения $T$ отрезка $[a, b]$ выполняется неравенство (3). (B [8] $M L$-интеграл вводился как сильньй интеграл Мак-Шейна.)

Легко видеть, что для этих интегралов справедливо утверждение, аналогичное теореме 1.2 .

ТЕОрема 2.1. Если функиия $f:[a, b] \rightarrow X$ ML-интегрируема на отрезке $[a, b]$, то она HL-интегрируема на отрезке $[a, b]$, при этом интегралы совпадают.

В терминах введенных интегралов теорема 1.4 может быть переформулирована следуюшим образом. 
Теорема 2.2. Пусть $Х$ - конечномерное банахово пространство. Имеют место следующие утверждения:

1) функиия $f:[a, b] \rightarrow X$ интегрируема по Хенстоку тогда и только тогда, когда она $H L$-интегрируема;

2) функиия $f:[a, b] \rightarrow X$ интегрируема по Мак-Шейну тогда и только тогда, когда она $M L$-интегрируема.

Далее мы рассмотрим случай, когда $X$ - бесконечномерное банахово пространство. Из результатов п. 3 будет следовать, что для такого пространства утверждение, аналогичное теореме 2.2, не вьполняется. Для получения этого результата нам понадобятся понятия $V B^{*}$-функции и $V B G^{*}$-функции, хорошо известные в действительном случае (см. $[9$, гл. VII $]$ ).

ОПредЕЛЕнИЕ 2.3. Функция $F:[a, b] \rightarrow X$ назьвается $V B^{*}$ - $у$ фкицией на множестве $E \subset[a, b]$, если существует число $M>0$ такое, что для любого набора неперекрьвающихся отрезков $\left\{\Delta_{i}\right\}_{i=1}^{n}$, концы которых принадлежат $E$, вьполняется неравенство

$$
\sum_{i=1}^{n} \omega\left(F, \Delta_{i}\right)<M
$$

Здесь

$$
\omega(F, P)=\sup _{t, s \in P}\|F(t)-F(s)\|
$$

- колебание функции $F$ на множестве $P$.

ОПРеДЕЛЕнИЕ 2.4. Функция $F:[a, b] \rightarrow X$ называется $V B G^{*}$ - функиией на множестве $E \subset[a, b]$, если $E$ представимо в виде счетного объединения множеств, на каждом из которых $F$ является $V B^{*}$-функцией.

Непосредственно из определений 2.3 и 2.4 и очевидного неравенства

$$
\omega(\|F\|, P) \leqslant \omega(F, P)
$$

вытекает

Лемма 2.1. Имеют место следующие утверждения:

1) если $F:[a, b] \rightarrow X$ является $V B^{*}$-функиией на множестве $E$, то $\|F\|$ также будет $V B^{*}$-функиией на $E$;

2) если $F:[a, b] \rightarrow X$ является $V B G^{*}$-функцией на множестве $E$, то $\|F\|$ также будет $V B G^{*}$-функиией на $E$.

Как и в действительном случае, первообразная $H L$-интегрируемой функции принадлежит классу $V B G^{*}$, что отражает следуюшая теорема.

Tеорема 2.3. Пусть функиия $f:[a, b] \rightarrow X$ является $H L$-интегрируемой с первообразной $F:[a, b] \rightarrow X$. Тогда $F$ является $V B G^{*}$-функиией на отрезке $[a, b]$. 
ДокАЗАТЕльство проведем, следуя [1], где уже разобран случай функций, принимающих действительные значения. Выберем положительную функцию $\delta(\xi)$ из условия $H L$-интегрируемости для $\varepsilon=1$ (без ограничения общности можно считать $\delta(\xi)<1$ ). Для каждого натурального $m$ найдем максимальное $l$ такое, что $a+l /(2 m) \leqslant b$. Положим для всех $m$ и $k, k \leqslant l+1$,

$$
E_{m}^{k}=\left\{t \in\left[a+\frac{k-1}{2 m}, a+\frac{k}{2 m}\right) \cap[a, b]:\|f(t)\| \leqslant m, \delta(t)>\frac{1}{m}\right\} .
$$

Очевидно, что $[a, b]=\bigcup_{m=1}^{\infty} \bigcup_{k=1}^{l+1} E_{m}^{k}$. Поэтому достаточно доказать, что $F$ является $V B^{*}$-функцией на каждом из множеств $E_{m}^{k}$. Фиксируем произвольные $m$ и $k$, для которых множество $E_{m}^{k}$ непусто. Рассмотрим произвольньй набор неперекрьвающихся отрезков $\left\{\left[c_{i}, d_{i}\right]\right\}_{i=1}^{n}$ с концами из $E_{m}^{k}$. Из построения множества $E_{m}^{k}$ видно, что подразбиение $\left\{\left(c_{i},\left[c_{i}, d_{i}\right]\right)\right\}_{i=1}^{n}$ является $\delta$-согласованньм по Хенстоку. Так как функция $F$ непрерьвна (по теореме 1.3 ), существуют точки $u_{i}, v_{i} \in\left[c_{i}, d_{i}\right]$ такие, что

$$
\left\|F\left(v_{i}\right)-F\left(u_{i}\right)\right\|=\omega\left(F,\left[c_{i}, d_{i}\right]\right)
$$

Принимая во внимание равенство (4), определение множества $E_{m}^{k}$ и $\delta$-согласованность по Хенстоку подразбиения, получаем

$$
\begin{aligned}
\sum_{i=1}^{n} \omega\left(F,\left[c_{i}, d_{i}\right]\right)= & \sum_{i=1}^{n}\left\|F\left(v_{i}\right)-F\left(u_{i}\right)\right\| \leqslant \sum_{i=1}^{n}\left\|F\left(v_{i}\right)-F\left(c_{i}\right)\right\|+\sum_{i=1}^{n}\left\|F\left(u_{i}\right)-F\left(c_{i}\right)\right\| \\
\leqslant & \sum_{i=1}^{n}\left\|F\left(u_{i}\right)-F\left(c_{i}\right)-f\left(c_{i}\right)\left(u_{i}-c_{i}\right)\right\|+\sum_{i=1}^{n}\left\|f\left(c_{i}\right)\right\|\left(u_{i}-c_{i}\right) \\
& +\sum_{i=1}^{n}\left\|F\left(v_{i}\right)-F\left(c_{i}\right)-f\left(c_{i}\right)\left(v_{i}-c_{i}\right)\right\|+\sum_{i=1}^{n}\left\|f\left(c_{i}\right)\right\|\left(v_{i}-c_{i}\right) \\
< & 1+m \cdot \frac{1}{m}+1+m \cdot \frac{1}{m}=4 .
\end{aligned}
$$

Следовательно, $F$ является $V B^{*}$-функцией на $E_{m}^{k}$, что и доказывает теорему.

3. Основной результат. Итак, цель этого пункта - доказательство следующей теоремы.

Теорема 3.1. Пусть $X$ - банахово пространство. Тогда следующие утверждения әквивалентны:

1) $X$ - конечномерное пространство;

2) функиия $f:[a, b] \rightarrow X$ интегрируема по Хенстоку тогда и только тогда, когда она $H L$-интегрируема;

3) функиия $f:[a, b] \rightarrow X$ интегрируема по Мак-Шейну тогда и только тогда, когда она $M L$-интегрируема.

Доказательство этой теоремы опирается на конструкцию, использованную в [10] для построения безусловно, но не абсолютно сходящегося ряда в бесконечномерном банаховом пространстве. 
Лемма 3.1. Пусть В - выпуклое тело в $\mathbb{R}^{n}$ с иентром в начале координат, $r$ натуральное число, не превосходящее $n$. Тогда на границе $B$ найдутся векторы $A_{1}, \ldots, A_{r} \in \mathbb{R}^{n}$ такие, что для любых чисел $\lambda_{1}, \ldots, \lambda_{r}$ выполняется

$$
\sum_{i=1}^{r} \lambda_{i} A_{i} \in \lambda B, \quad \text { əде } \quad \lambda^{2}=\left(2+\frac{r(r-1)}{n}\right) \sum_{i=1}^{r} \lambda_{i}^{2} .
$$

Через $\lambda В$ обозначено множество $\{\lambda x, x \in B\}$.

Доказательство леммы 3.1 можно найти в [10].

ЛЕмма 3.2. Пусть $X$ - бесконечномерное банахово пространство, $c_{1}, \ldots, c_{r}-$ произвольные положительные числа. Тогда найдутся векторы $x_{1}, \ldots, x_{r}$ пространства $X$ такие, что

$$
\left\|x_{i}\right\|^{2}=c_{i}, \quad 1 \leqslant i \leqslant r,
$$

$u$ для любых чисел $\theta_{1}, \ldots, \theta_{r}$, удовлетворяющих условию $\left|\theta_{i}\right| \leqslant 1$, выполняется неравенство

$$
\left\|\sum_{i=1}^{r} \theta_{i} x_{i}\right\|^{2} \leqslant 3 \sum_{i=1}^{r} c_{i} .
$$

ДокАЗАТЕЛЬСтво. В силу бесконечномерности пространства $X$ для каждого натурального числа $n$ найдутся линейно независимые векторы $z_{1}, \ldots, z_{n}$ пространства $X$. Выберем $n=r(r-1)$. Обозначим через $B$ множество векторов $z$, представимых в виде

$$
z=\sum_{i=1}^{n} \mu_{i} z_{i}
$$

где $\mu_{i}$ - числа такие, что $\|z\| \leqslant 1$. Тогда по лемме 3.1 на границе множества $B$ можно найти векторы $A_{1}, \ldots, A_{r}$ такие, что для любьх чисел $\lambda_{1}, \ldots, \lambda_{r}$ справедливо

$$
\sum_{i=1}^{r} \lambda_{i} A_{i} \in \lambda B, \quad \text { где } \lambda^{2}=3 \sum_{i=1}^{r} \lambda_{i}^{2} .
$$

Так как для всех $i=1, \ldots, r$ векторы $A_{i}$ лежат на границе $B$, то

$$
\left\|A_{i}\right\|=1 \text {. }
$$

Поскольку $\|z\| \leqslant 1$ для всех $z \in B$, из (6) следует, что

$$
\left\|\sum_{i=1}^{r} \lambda_{i} A_{i}\right\|^{2} \leqslant 3 \sum_{i=1}^{r} \lambda_{i}^{2} .
$$

Выберем $x_{i}=\sqrt{c_{i}} A_{i}$. Тогда в силу (7) $\left\|x_{i}\right\|^{2}=c_{i}$. Для доказательства неравенства (5) достаточно применить неравенство $(8)$, положив $\lambda_{i}=\theta_{i} \sqrt{c_{i}}$, и воспользоваться тем, что $\left|\theta_{i}\right| \leqslant 1$ :

$$
\left\|\sum_{i=1}^{r} \theta_{i} x_{i}\right\|^{2}=\left\|\sum_{i=1}^{r} \theta_{i} \sqrt{c_{i}} A_{i}\right\|^{2} \leqslant 3 \sum_{i=1}^{r} \theta_{i}^{2} c_{i} \leqslant 3 \sum_{i=1}^{r} c_{i} .
$$

Докажем теперь основную техническую лемму. 
Лемма 3.3. Пусть $X$ - бесконечномерное банахово пространство. Тогда существует функиия $f:[a, b] \rightarrow X$, обладающая следующими свойствами:

1) $f$ интегрируема по Мак-Шейну;

2) ее первообразная $F:[a, b] \rightarrow X$ не является $V B G^{*}$-функиией.

ДоКАЗАТЕЛЬСТво. Предположим для простоты, что $[a, b]=[0,1]$. Пусть $C-$ канторово множество, $\left(a_{i}^{r}, b_{i}^{r}\right), r \geqslant 0,1 \leqslant i \leqslant 2^{r}$,- смежные к $C$ интервалы длины $3^{-r-1}$; $d_{i}^{r}$ - середины интервалов $\left(a_{i}^{r}, b_{i}^{r}\right)$.

По лемме 3.2 для каждого $r$ построим векторы $x_{1}^{r}, x_{2}^{r}, \ldots, x_{2^{r}}^{r} \in X$ такие, что

$$
\left\|x_{i}^{r}\right\|=\frac{1}{2^{r}}, \quad 1 \leqslant i \leqslant 2^{r},
$$

и для любых чисел $\theta_{1}^{r}, \theta_{2}^{r}, \ldots, \theta_{2^{r}}^{r}$, удовлетворяюших условию $\left|\theta_{i}^{r}\right| \leqslant 1$, вьполняется неравенство

$$
\left\|\sum_{i=1}^{2^{r}} \theta_{i}^{r} x_{i}^{r}\right\|^{2} \leqslant 3 \sum_{i=1}^{2^{r}} \frac{1}{4^{r}}
$$

которое можно записать в виде

$$
\left\|\sum_{i=1}^{2^{r}} \theta_{i}^{r} x_{i}^{r}\right\|^{2} \leqslant \frac{3}{2^{r}}
$$

Определим функцию $f:[0,1] \rightarrow X$ следующим образом:

$$
f(t)=\left\{\begin{array}{rl}
0, & \text { если } t \in C \text { или } t=d_{i}^{r}, \\
2 \cdot 3^{r} x_{i}^{r}, & \text { если } t \in\left(a_{i}^{r}, d_{i}^{r}\right), \\
-2 \cdot 3^{r} x_{i}^{r}, & \text { если } t \in\left(d_{i}^{r}, b_{i}^{r}\right),
\end{array} \quad r \geqslant 0, \quad 1 \leqslant i \leqslant 2^{r} .\right.
$$

Покажем, что построенная функция $f$ интегрируема по Мак-Шейну и для любого $s \in C$ интеграл по отрезку $[0, s]$ равен 0 . Введем следующие обозначения:

$$
\begin{aligned}
& U_{i}^{r}=[0, s] \cap\left(a_{i}^{r}, b_{i}^{r}\right) \backslash\left\{d_{i}^{r}\right\}, \quad U_{R}=\bigcup_{r=0}^{R} \bigcup_{i=1}^{2^{r}} U_{i}^{r}, \\
& U=\bigcup_{R=0}^{\infty} U_{R}, \quad V=[0, s] \cap C \cup\left(\bigcup_{r=0}^{\infty} \bigcup_{i=1}^{2^{r}}\left\{d_{i}^{r}\right\}\right) .
\end{aligned}
$$

$\Phi$ иксируем произвольное $\varepsilon>0$. Номер $R$ и действительное число $\rho$ выберем так, чтобы

$$
\begin{gathered}
2^{R}>\frac{16}{\varepsilon^{2}}, \\
\rho \cdot 3^{R+1}<\frac{\varepsilon}{4} .
\end{gathered}
$$

Положительную функцию $\delta(\xi)$ зададим следующим образом:

$$
\begin{array}{ll}
\delta(\xi)=\min \left\{\left|\xi-a_{i}^{r}\right|,\left|\xi-b_{i}^{r}\right|,\left|\xi-d_{i}^{r}\right|\right\}, & \text { если } \xi \in U_{i}^{r}, r \geqslant 0,1 \leqslant i \leqslant 2^{r}, \\
\delta(\xi)=\rho, & \text { если } \xi \in V .
\end{array}
$$


Рассмотрим произвольное $\delta$-согласованное по Мак-Шейну разбиение $T$ отрезка $[0, s]$. Ясно, что $U \cup V=[0, s]$ и $f(\xi)=0$ для всех $\xi \in V$. Поэтому

$$
\sum_{T} f\left(\xi_{k}\right)\left|\Delta_{k}\right|=\sum_{\xi_{k} \in U} f\left(\xi_{k}\right)\left|\Delta_{k}\right|
$$

Можно считать, что каждьй отрезок $\Delta_{k}$ обладает следующим свойством: он либо попадает в множество $\bar{U}_{R}$ целиком, либо не пересекается с $U_{R}(\bar{P}$ обозначает замыкание множества $P$ ). Если это не так, разобьем каждый отрезок $\Delta_{k}$ на конечное число отрезков, обладающих указанным свойством, и сопоставим каждому из этих отрезков одну и ту же точку $\xi_{k}$. При этом свойство $\delta$-согласованности по Мак-Шейну, очевидно, не нарушится. В силу последнего замечания имеем

$$
\left\|\sum_{\xi_{k} \in U} f\left(\xi_{k}\right)\left|\Delta_{k}\right|\right\| \leqslant\left\|\sum_{\Delta_{k} \subset \bar{U}_{R}} f\left(\xi_{k}\right)\left|\Delta_{k}\right|\right\|+\left\|\sum_{\Delta_{k} \cap U_{R}=\varnothing} f\left(\xi_{k}\right)\left|\Delta_{k}\right|\right\| .
$$

Оценим каждое слагаемое. Принимая во внимание определение функции $f$, выбор функции $\delta(\xi)$ такой, как в (14), а также вспоминая, что $s \in C$, можно выбрать числа $\theta_{i}^{r}$, $0 \leqslant r \leqslant R, 1 \leqslant i \leqslant 2^{r}$, так что $\left|\theta_{i}^{r}\right| \leqslant 2 \rho$ и

$$
\sum_{\Delta_{k} \subset \bar{U}_{R}} f\left(\xi_{k}\right)\left|\Delta_{k}\right|=\sum_{r=0}^{R} \sum_{i=1}^{2^{r}} 2 \cdot 3^{r} \theta_{i}^{r} x_{i}^{r}
$$

Применяя соотношения (9) и (12), получим

$$
\left\|\sum_{\Delta_{k} \subset \bar{U}_{R}} f\left(\xi_{k}\right)\left|\Delta_{k}\right|\right\| \leqslant \sum_{r=0}^{R} 2 \cdot 3^{r} \sum_{i=1}^{2^{r}} 2 \rho\left\|x_{i}^{r}\right\|=2 \rho\left(3^{R+1}-1\right)<\frac{\varepsilon}{2} .
$$

Оценим, наконец, второе слагаемое в (16). Принимая во внимание выбор функции $\delta(\xi)$ такой, как в $(13)$, найдем числа $\theta_{i}^{r}, r \geqslant R, 1 \leqslant i \leqslant 2^{r}$, такие, что $\left|\theta_{i}^{r}\right| \leqslant 1$ и

$$
\sum_{\Delta_{k} \cap U_{R}=\varnothing} f\left(\xi_{k}\right)\left|\Delta_{k}\right|=\sum_{r=R+1}^{\infty} \sum_{i=1}^{2^{r}} \theta_{i}^{r} x_{i}^{r}
$$

Следовательно, применяя соотношения (10) и (11), заключаем, что

$$
\left\|\sum_{\Delta_{k} \cap U_{R}=\varnothing} f\left(\xi_{k}\right)\left|\Delta_{k}\right|\right\| \leqslant \sum_{r=R+1}^{\infty}\left\|\sum_{i=1}^{2^{r}} \theta_{i}^{r} x_{i}^{r}\right\| \leqslant \sum_{r=R+1}^{\infty} \sqrt{3} \cdot 2^{-r / 2}<2 \cdot 2^{-R / 2}<\frac{\varepsilon}{2} .
$$

Объединяя (15)-(18), приходим к неравенству

$$
\left\|\sum_{T} f\left(\xi_{i}\right)\left|\Delta_{k}\right|\right\|<\varepsilon
$$


Тем самым, мы проверили интегрируемость по Мак-Шейну функции $f$ и показали, что ее первообразная обрашается в 0 в каждой точке $s \in C$. В силу конечной аддитивности неопределенного интеграла Мак-Шейна первообразная функции $f$ имеет вид

$$
F(t)= \begin{cases}0, & \text { если } t \in C, \\ 2 \cdot 3^{r}\left(t-a_{i}^{r}\right) x_{i}^{r}, & \text { если } t \in\left(a_{i}^{r}, d_{i}^{r}\right], r \geqslant 0,1 \leqslant i \leqslant 2^{r}, \\ -2 \cdot 3^{r}\left(t-b_{i}^{r}\right) x_{i}^{r}, & \text { если } t \in\left(d_{i}^{r}, b_{i}^{r}\right), r \geqslant 0,1 \leqslant i \leqslant 2^{r} .\end{cases}
$$

Осталось доказать, что функция $F$ не является $V B G^{*}$-функцией. Пусть это не так. Тогда из утверждения 2) леммы 2.1 следует, что $\|F\|$ является $V B G^{*}$-функцией. Для $\|F\|$ имеем следующее представление:

$$
\|F(t)\|= \begin{cases}0, & \text { если } t \in C, \\ 3^{r}\left(t-a_{i}^{r}\right) / 2^{r-1}, & \text { если } t \in\left(a_{i}^{r}, d_{i}^{r}\right], r \geqslant 0,1 \leqslant i \leqslant 2^{r}, \\ -3^{r}\left(t-b_{i}^{r}\right) / 2^{r-1}, & \text { если } t \in\left(d_{i}^{r}, b_{i}^{r}\right), r \geqslant 0,1 \leqslant i \leqslant 2^{r} .\end{cases}
$$

Известно (см. [11, с. 224]), что эта функция не является $V B G^{*}$-функцией. Полученное противоречие доказывает лемму.

ДокАЗАТЕЛЬСтво теОРЕмы 3.1. Импликации 1$) \Longrightarrow 2$ ), 1) $\Longrightarrow 3$ ) немедленно вытекают из теоремы 2.2 .

$2) \Longrightarrow 1$ ). Пусть $X$ бесконечномерно. Тогда в силу леммы 3.3 существует интегрируемая по Мак-Шейну функция $f:[a, b] \rightarrow X$, первообразная которой не являтся $V B G^{*}$-функцией. По теореме 1.2 функция $f$ интегрируема по Хенстоку, что по условию предполагает $H L$-интегрируемость. Но в силу теоремы 2.3 первообразная функции $f$ в этом случае должна быть $V B G^{*}$-функцией. Полученное противоречие доказывает конечномерность пространства $X$.

$3) \Longrightarrow 1$ ). Доказательство аналогично предыдущему. Вместо теоремы 1.2 необходимо воспользоваться теоремой 2.1 .

В работе [3] сформулировано достаточное условие эквивалентности интеграла Хенстока и $H L$-интеграла, использующее понятие $A S$-оператора. Обозначим через $X^{*}$ пространство линейных непрерьвных функционалов над $X$.

ОпредЕЛЕниЕ 3.1. Линейный непрерывньй оператор $T: X \rightarrow X$ называется $A S$ оператором, если существует положительное число $M$ такое, что для любого конечного набора векторов $x_{1}, \ldots, x_{n}$ из $X$ вьполняется неравенство

$$
\sum_{k=1}^{n}\left\|T x_{k}\right\| \leqslant M \sup _{\substack{x^{*} \in X^{*} \\\left\|x^{*}\right\| \leqslant 1}} \sum_{k=1}^{n}\left|x^{*}\left(x_{k}\right)\right| .
$$

TЕОРема 3.2 (см. [3]). Если пространство X таково, что единичный оператор является $A S$-оператором, то интеграл Хенстока и $H L$-интеграл эквивалентны.

Поскольку в конечномерном пространстве единичный оператор является $A S$-оператором, лемма Сакса-Хенстока (теорема 1.4) может быть получена как следствие теоремы 3.2 .

Автор, пользуясь случаем, благодарит профессора В. А. Скворцова за помощь в работе над статьей. 


\section{СПИСОК ЦИТИРОВАННОЙ ЛИТЕРАТУРЫ}

[1] Gordon R. Equivalence of the generalized Riemann and restricted Denjoy integral // Real Anal. Exchange. 1986/87. V. 12. № 2. P. 551-574.

[2] McShane E. J. Unified Integration. New York: Acad. Press, 1983.

[3] Cao S. S. The Henstock integral for Banach-valued functions // Southeast Asian Bull. Math. 1992. V. 16. № 1. P. 35-40.

[4] Cao S. S. Banach-valued Henstock integration // Real Anal. Exchange. 1993/94. V. 19. № 1. P. 34 .

[5] Gordon R. The McShane integral of Banach-valued functions // Illinois J. Math. 1990. V. 34. P. 557-567.

[6] Pfeffer W. The Riemann Approach to Integration. Cambridge: Cambridge Univ. Press, 1993.

[7] Mawhin J. Generalized multiple Perron integrals and the Green-Goursat theorem for differentiable vector fields // Czechoslovak Math. J. 1981. V. 31(106). № 4. P. 614-632.

[8] Congxin W., Xiaobo Y. A Riemann-type definition of the Bochner integral // J. Math. Study. 1994. V. 27. № 1. P. 32-36.

[9] Сакс С. Теория интеграла. М.: ИЛ, 1949.

[10] Dvoretzky A., Rogers C. A. Absolute and unconditional convergence in normed linear spaces // Proc. Nat. Acad. Sci. USA. 1950. V. 36. № 3. P. 192-197.

[11] Ene V. Real Functions - Current Topics. Lecture Notes in Math. V. 1603. Berlin: Springer, 1995.

Московский государственный университет им. М.В. Ломоносова

Поступило

E-mail: asolodov@nw.math.msu.su

05.05.97

Исправленный вариант

30.07.98 